Research Article

\title{
The Study of a Mathematical Model in Information Acquisition and Disclosure
}

\author{
Ce Huang \\ School of Public Finance and Taxation, Southwestern University of Finance and Economics, 555 Liutai Avenue, \\ Wenjiang, Chengdu, Sichuan 611130, China
}

Correspondence should be addressed to Ce Huang; huangce@swufe.edu.cn

Received 7 October 2014; Accepted 23 November 2014

Academic Editor: Honglei Xu

Copyright (C) 2015 Ce Huang. This is an open access article distributed under the Creative Commons Attribution License, which permits unrestricted use, distribution, and reproduction in any medium, provided the original work is properly cited.

A mathematical model involving a decision maker and an expert is investigated. Through analyzing the model, we obtain several results on the expert's information acquisition and disclosure strategy. When withholding information is costly to the expert, in equilibrium, an expert with a higher withholding cost acquires less information but discloses more acquired information. We also examine which expert is optimal to the decision maker among a group of experts with different costs of withholding information.

\section{Introduction}

In many situations, a decision maker needs to make a decision but does not know which decision is the best one. For example, an investor may not know the future performance of companies in the stock market and is uncertain about which stock is the best. A patient does not have knowledge about the most cost-effective treatment for the health concern. Facing lack of information, the decision maker often looks for advice from investment consultants or doctors, who are experts and are better informed.

However, the interest of the expert is often different from that of the decision maker. In other words, the decision preferred by the expert is different from the one preferred by the decision maker. In our previous examples, an investment consultant may not be interested in picking the best stock. Instead, the consultant may want the investor to buy some particular stocks, perhaps because these stock companies will pay the consultant. Doctors often receive bonuses from expensive medical procedures, which might be unnecessary for patients with mild diseases. Due to this conflict of interests between experts and decision makers, experts often withhold information from decision makers.

We analyze a game between a decision maker (hereafter DM) and an expert (we use "she" to denote DM and "he" to denote the expert throughout this paper). DM needs to take a decision. The best decision of DM depends on an uncertain state of the world (hereafter the state). DM has no information about the state but the expert, through exerting costly effort to acquire information, can observe the state with some probability. The probability of observing the state is increasing in his effort. DM and the expert have different preferences. DM's best decision is equal to the state but the expert prefers a decision strictly higher than the state. Upon observing the state, the expert can disclose the observation to DM or withhold the information. We analyze the perfect Bayesian Nash equilibrium of the game between DM and the expert. In equilibrium, the expert decides how much effort to spend in information acquisition and what range of acquired information to disclose to DM, and DM chooses which decision to be taken when the expert does not disclose information.

We consider a situation where withholding information is costly to the expert. This is because the expert may feel guilty if he withholds information from DM. The cost of withholding information reflects the loss in the expert's utility caused by guilty feelings. An alternative explanation for the cost is that DM may discover the withholding behavior of the expert. As a result, DM will doubt the trustworthy of the expert and refuse seeking advice from the expert in the future. The cost of withholding information to the expert reflects the expert's loss in his future revenue due to losing 
clients. Of course, different experts have different costs of withholding information. This is because different experts have different moral standards or have different concerns for future revenues.

We study how the expert's equilibrium information acquisition and disclosure strategy changes as the expert's withholding cost increases. We also study how the expected utility of DM changes. These questions are investigated under two different utility functions of the expert. Under both utility functions, the expert exerts less effort in acquiring information and discloses a greater proportion of collected information as withholding cost increases. If the expert prefers the decision to be as high as possible regardless of the state, the expert's effort in acquiring information quickly decreases as withholding cost increases. As a result, the likelihood that the expert can obtain information quickly decreases. Although the expert discloses a greater proportion of acquired information to DM, DM gets less information and gets worse off. Alternatively, if the ideal decision of the expert is higher than that of DM by a constant distance, the expert's effort in information acquisition slowly decreases as withholding cost increases. DM receives more information and gets better off.

This paper is related to a large literature on the strategic transmission of verifiable information between a decision maker and an expert. See Grossman [1], Grossman and Hart [2], Milgrom [3], and Milgrom and Roberts [4] for early classic studies on this topic. When information is verifiable, DM can check the truthfulness of the expert's disclosed information. Therefore, it is assumed in this literature that the expert cannot disclose information that is different from the observed state. However, the expert can withhold the information. These papers mentioned above find that the expert will not withhold information in equilibrium. To see why, suppose that the expert withholds information when some states are observed. When the expert does not disclose information, DM infers that the state is at the average value among these states. This implies that the expert should disclose the observed state that is higher than the average value, because disclosing the state induces DM to take a higher decision. The expert is better off by withholding information only when the observed state is lower than the average. Knowing this, DM adjusts her inference of the state downwards when the expert does not disclose information. Again, the expert who observes a state higher than the new inference of DM is better off by disclosing information. We can repeat the same argument and show that the expert withholds information only when he observes the lowest possible state. Knowing this, DM can infer the state when the expert does not disclose information. Therefore, there is no information withholding in equilibrium.

It is assumed in Shin $[5,6]$ that the expert fails to obtain information about the state with a positive probability. When the expert does not disclose information, DM takes into account the possibility that the expert does not obtain information. In equilibrium, the expert will withhold information when some states are observed.

In Che and Kartik [7], in order to obtain information about the state, the expert needs to exert costly effort. When more effort is exerted, the expert will more likely obtain the information. It is found that when the expert has a prior belief about the state different from that of DM, the expert will exert more effort in acquiring information. It is established that DM can receive a higher expected utility from consulting an expert with a slightly different prior belief about the state, compared to the utility from consulting an expert with the same prior belief. In Xu and Suen [8], a model similar to the one in Che and Kartik [7] is considered. In Xu and Suen [8], withholding information by the expert may be detected by $\mathrm{DM}$ and the expert will be punished. It is found that, under a larger punishment on withholding, the expert may acquire less information and disclose less acquired information to DM. The current paper studies an issue similar to the one in $\mathrm{Xu}$ and Suen [8]. However, the current paper considers a case where the state can take a continuum of possible values, while there are only two possible states in $\mathrm{Xu}$ and Suen [8]. The current paper is different from Xu and Suen [8] also in predictions. In the current paper, the expert discloses more acquired information under a larger punishment on withholding information, while, in $\mathrm{Xu}$ and Suen [8], the expert discloses less acquired information.

The paper is organized as follows. Section 2 builds a model of information acquisition and transmission between an expert and a decision maker. Section 3 characterizes the relationship between the expert's withholding cost and the effort in obtaining information. Also the relationship between withholding cost and the range of disclosed information is characterized. Section 4 examines which one is the best to DM in a group of experts with different costs of withholding information. Section 5 considers a model where the utility function of the expert takes a different form. Section 6 concludes and discusses future work.

\section{Model}

A decision maker needs to make a decision, modeled as a choice of a real number in the interval $[0,1]$. Her utility from decision $a \in[0,1]$ depends on a variable $\theta$, which is called the state of the world. Assume that her utility is

$$
u_{\mathrm{DM}}(a, \theta)=-(a-\theta)^{2} .
$$

Apparently, DM's utility is higher when $a$ is closer to $\theta$. This reflects DM's desire to take a decision as close to the state as possible.

However, DM has no information about $\theta$. We assume that $\theta$ is uniformly distributed on $[0,1]$. In order to get more information about the state and make a better decision, DM consults an expert who can potentially observe $\theta$.

Initially, the expert does not have any information about $\theta$. However, the expert can exert effort to acquire information about $\theta$, so that $\theta$ can be observed with some probability. The more effort the expert exerts, the more likely $\theta$ will be observed. Exerting effort is costly to the expert. We assume that, in order to observe $\theta$ with probability $p$, the expert needs to exert effort that costs him $c(p)$, where $c(p)$ strictly increases in $p$. 
The expert's preference is different from that of DM. In the benchmark case, we assume that when the decision taken by $\mathrm{DM}$ is $a$ and the state is $\theta$, the utility of the expert is

$$
u_{E}(a, \theta)=a \text {. }
$$

The expert's utility only depends on the level of DM's decision and is independent of the state. Therefore, the expert is not concerned about whether the decision is appropriate for the state or not. Instead, the expert only cares about the level of the decision. We will consider another utility function of the expert in Section 5.

When the expert observes $\theta$, we assume that the expert cannot lie to DM by reporting that the state is $\theta^{\prime}$, which is different from the observed $\theta$. However, the expert can say that he does not observe the state. This is because the expert does not observe the state for sure and DM does not know whether the expert observes $\theta$ or not. We call this behavior "withholding information." The expert who observes that the state is $\theta$ can report either " $\theta$," the state he observes, or " $\Phi$." Here $\Phi$ stands for "I do not know about the state." When the expert does not observe the state, we assume that the expert can only report " $\Phi$." We assume that when the expert observes that the state is $\theta$, reporting $\theta$ is costless to the expert while reporting " $\Phi$ " is costly. We assume that the cost is $x \geq 0$.

When the expert reports " $\Phi$ " (hereafter "the expert does not disclose information"), DM cannot tell whether the expert indeed does not observe the state or is just withholding information. When making a decision, DM takes into account the probability that the expert withholds information.

The equilibrium concept is perfect Bayesian Nash equilibrium (hereafter "the equilibrium"). It consists of three components: the expert's strategy in how much effort to exert in acquiring information about the state and which states to be disclosed to DM after observing the state; DM's strategy in what decision to be taken when the expert does not disclose information; and DM's belief about the state when the expert does not disclose information. To be an equilibrium, these strategies and belief are such that, given DM's strategy, the expert's strategy maximizes his expected utility, net of the expected cost and given DM's belief about the state, DM's strategy maximizes her expected utility; DM's belief is derived from the expert's strategy using Bayes' rule.

Denote the decision taken by DM by $a_{\phi}$ when the expert does not disclose information. We consider whether the expert will disclose information when the observed state is $\theta$. If the expert discloses the information, DM believes that the state is $\theta$ and takes a decision that gives DM the highest utility, which is $\theta$. The utility of the expert by disclosing information is $\theta$. If the expert withholds the information, DM will take decision $a_{\phi}$. The expert's utility, net of the cost of withholding information, is $a_{\phi}-x$. So the expert will disclose information when the observed state satisfies

$$
\theta \geq a_{\phi}-x
$$

Now we consider how much effort the expert will exert in information acquisition, given DM's decision in the event of nondisclosure, $a_{\phi}$, and the expert's optimal information disclosing strategy derived above. Note that when deciding how much effort to exert in acquiring information, the expert has not observed the state yet. When the expert exerts effort that costs him $c(p)$, he will observe the state with probability $p$ and will not observe the state with probability $1-p$. In the former case, when the observed state is less than $a_{\phi}-x$, the expert will withhold the information from DM and receive utility (net of the withholding cost) $a_{\phi}-x$. When the observed state is greater than $a_{\phi}-x$, the expert will disclose the information to DM and receive a utility equal to the observed state. If the expert does not observe the state, he will report $\Phi$ to DM and receive utility $a_{\phi}$. Therefore, the expert's expected utility when he exerts effort in information acquisition that costs $\operatorname{him} c(p)$ is

$$
\begin{aligned}
& p\left[\left(a_{\phi}-x\right)^{2}+\left(1-a_{\phi}+x\right) * \frac{\left(1+a_{\phi}-x\right)}{2}\right] \\
& \quad+(1-p) a_{\phi}-c(p)
\end{aligned}
$$

where $\left(1+a_{\phi}-x\right) / 2$ in the square bracket is the expected value of $\theta$ conditional on being greater than $a_{\phi}-x$.

In order to maximize the expected utility, the expert will exert effort in acquiring information such that the probability $p$ that the expert obtains information satisfies

$$
c^{\prime}(p)=l\left(a_{\phi}, x\right)
$$

where $l(a, x) \equiv\left[1+(a-x)^{2}\right] / 2-a$.

Through straightforward calculation, we have the following result regarding function $l(a, x)$.

Lemma 1. When $x \leq a \leq 1, l(a, x)$ strictly decreases in $a$ and strictly decreases in $x$.

Intuitively, the incentive of the expert to acquire information is from the difference between the expert's expected utility when information is successfully obtained and his expected utility when failing to obtain information. As $a_{\phi}$ increases, the difference between these two expected utilities decreases. Thus, the incentive of the expert to acquire information decreases. As $x$ increases, the expert's expected utility when information is successfully obtained decreases while the expected utility when failing to obtain information does not change. Thus, the expert's incentive to acquire information decreases.

As the next step, we characterize DM's posterior belief about the state when the expert does not disclose information. The belief is derived from the expert's strategy in information acquisition and disclosure using Bayes' rule.

Suppose that the expert exerts effort to acquire information, and the effort exerted costs the expert $c(p)$. When information about the state is obtained, the expert withholds information if and only if the observed $\theta$ is lower than a cutoff value $k$ (given the expert's utility function, any equilibrium strategy in information disclosure must have this "cutoff" property). Under this strategy of the expert, with probability $1-p$ the expert will fail to observe the state, and with probability $p k$, the expert successfully observes the state and 
the observed state is less than $k$. In the latter case, the expert will withhold information. Conditional on that the expert does not disclose information to DM, by Bayes' rule, the probability that the expert withholds information is $p k /[(1-$ $p)+p k]$, and the expert is genuinely uninformed about the state with the complementary probability. The state follows a uniform distribution on $[0, k]$ when the expert withholds information and follows a uniform distribution on $[0,1]$ when the expert is genuinely uninformed about the state. Therefore, when receiving no information from the expert, DM's posterior belief is that the state follows the distribution function:

$$
\begin{aligned}
F & (\theta \mid \text { No disclosure }) \\
& = \begin{cases}\frac{p k}{1-p+p k} \frac{\theta}{k}+\frac{1-p}{1-p+p k} \theta, & \text { if } \theta \leq k, \\
\frac{p k}{1-p+p k}+\frac{1-p}{1-p+p k} \theta, & \text { if } \theta>k .\end{cases}
\end{aligned}
$$

Compared to DM's prior belief about the state, DM's posterior belief about the state when the expert does not disclose information features a higher probability density at $\theta \leq k$ and a lower probability density at $\theta>k$. Intuitively, when the expert does not disclose information, DM thinks that the expert might withhold information from DM. Because the expert withholds information when the state is relatively low, DM thinks that the state is more likely to be low and less likely to be high.

Under the posterior belief, DM's optimal decision $a_{\phi}$ when the expert does not disclose information satisfies

$$
a_{\phi} \in \underset{a \in[0,1]}{\operatorname{argumax}} E_{\theta}\left[-(a-\theta)^{2} \mid F(\cdot \mid \text { No disclosure })\right],
$$

from which we can solve $a_{\phi}$ as

$$
a_{\phi}=h(p, k)
$$

where $h(p, k) \equiv\left[p k^{2} /(1-p+p k)+(1-p) /(1-p+p k)\right] / 2$. We have the following result regarding function $h(p, k)$.

Lemma 2. $h(p, k)$ strictly decreases in $p$ when $k<1$. When $k$ is small, $h(p, k)$ strictly decreases in $k$; when $k$ is large, $h(p, k)$ strictly increases in $k$.

Proof. Note that $h(p, k)$ is the average of $k / 2$ and $1 / 2$ with the weight on $k / 2$ being $p k /(1-p+p k)$. When $p$ increases, the weight on $k / 2$ increases, which implies that $h(p, k)$ decreases when $k<1$.

To see how $h(p, k)$ changes as $k$ increases, note that

$$
\frac{\partial h(p, k)}{\partial k}=\frac{1}{2} \frac{p k}{1-p+p k}\left[1-\frac{1-k}{k} \frac{1-p}{1-p+p k}\right] \text {. }
$$

It can be seen that if $0<p<1$, there exists a cutoff value $0<\widehat{k}<1$ such that $\partial h(p, k) / \partial k<0$ when $k<\widehat{k}$ and $\partial h(p, k) / \partial k>0$ when $k>\widehat{k}$.

Intuitively, when the probability that the expert obtains information is higher, it is more likely that the expert is withholding information when the expert does not disclose information. Given the value of the state is relatively low when the expert withholds information, DM's expectation about the state is lower. Therefore, her optimal decision $h(p, k)$ decreases.

When the cutoff value of expert's disclosing strategy increases, there are two conflicting effects. On the one hand, with a higher cutoff value and more states under which the expert will withhold information, DM thinks that the probability that the expert withholds information is higher when the expert does not disclose information. Therefore, DM's expectation of the state decreases and her optimal decision $h(p, k)$ also decreases. On the other hand, a higher cutoff value implies that the mean value of the state increases when the expert withholds information. DM's expectation of the state increases and her optimal decision $h(p, k)$ also increases. When $k$ is small, the first effect dominates and $h(p, k)$ decreases in $k$. However, when $k$ is large, the second effect dominates and $h(p, k)$ increases in $k$.

A perfect Bayesian Nash equilibrium can be represented as a tuple $\left\{a_{\phi}^{*}, p^{*}, k^{*}\right\}$ such that $c^{\prime}\left(p^{*}\right)=l\left(a_{\phi}^{*}, x\right) ; k^{*}=a_{\phi}^{*}-x$; $a_{\phi}^{*}=h\left(p^{*}, k^{*}\right)$. We assume that $x$ is small enough such that, in equilibrium, the expert exerts some effort in information acquisition; that is, $p^{*}>0$. Apparently, $a_{\phi}^{*}, p^{*}$, and $k^{*}$ are all functions of $x$. We suppress the dependence of these equilibrium variables on $x$ in order to keep notations simple.

\section{Equilibrium Characterization}

In this section, we address the following question: under a greater cost of withholding information, will the expert exert more effort in information acquisition and withhold less acquired information from DM in the equilibrium? In other words, when $x$ increases, will $p^{*}$ increase and $k^{*}$ decrease?

We use numerical simulation to get the relationship between $x$ and equilibrium variables, $p^{*}, k^{*}$, and $a_{\phi}^{*}$. As shown in Figure 1, when $x$ increases, $p^{*}$ decreases, $k^{*}$ decreases, and $a_{\phi}^{*}$ increases. Under a greater cost of withholding information, the expert exerts less effort in information acquisition and withholds less acquired information. We have the following result.

Proposition 3. As $x$ increases, $p^{*}$ decreases, $k^{*}$ decreases, and $a_{\phi}^{*}$ increases.

To see why the above relationship holds, suppose that $p^{*}$ does not change when $x$ increases. This allows us to see how $a_{\phi}^{*}$ and $k^{*}$ change. For a fixed $p^{*}, a_{\phi}^{*}$ and $k^{*}$ can be characterized as the solution to equations $k=a_{\phi}-x$ and $a_{\phi}=h\left(k, p^{*}\right)$. As shown in Figure 2, when $x$ increases, the curve $k=a_{\phi}-x$ shifts upward and the curve $a_{\phi}=$ $h\left(k, p^{*}\right)$ remains unchanged. Therefore, $a_{\phi}^{*}$ increases and $k^{*}$ decreases, implying that $l\left(a_{\phi}^{*}, x\right)$ decreases. Therefore, the expert's incentive to acquire information decreases, implying that $p^{*}$ decreases.

Intuitively, as $x$ increases, the expert withholds less information from DM. $k^{*}$ decreases. This implies that the 


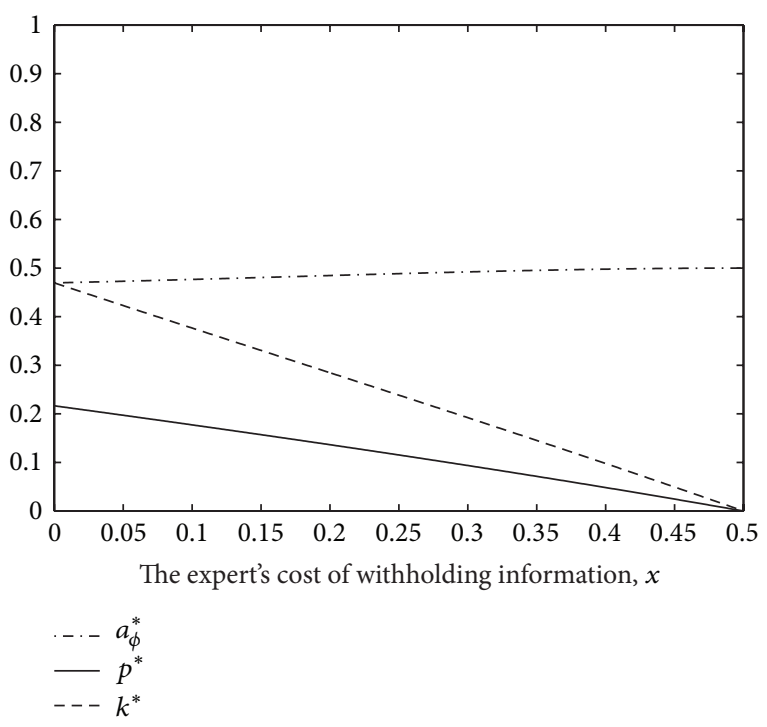

FIgURE 1: How equilibrium variables change as $x$ changes.

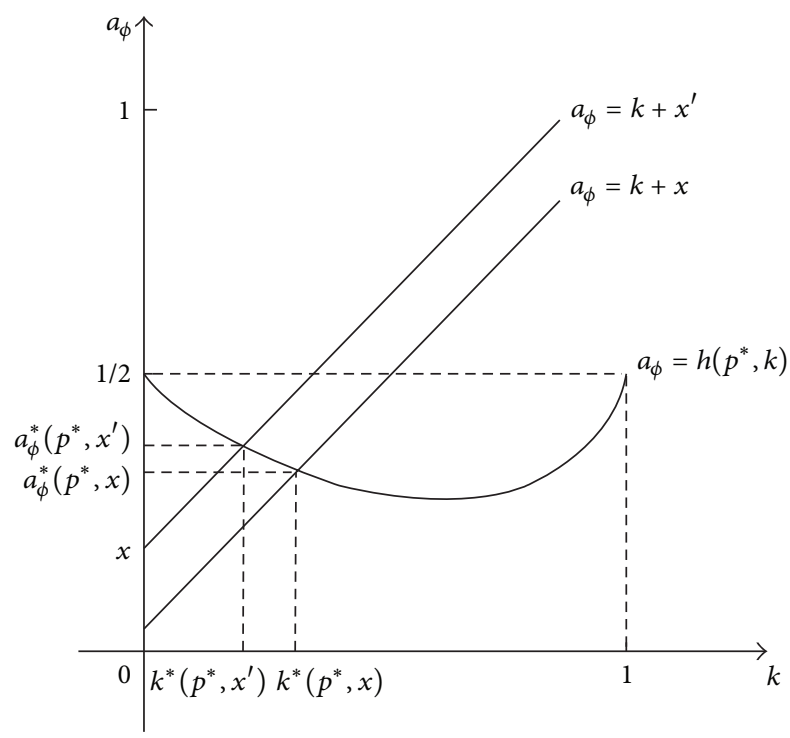

FIgURE 2: A higher $x$ implies a lower $k$ and a higher $a$.

likelihood that the expert withholds information decreases. As a result, DM thinks that it is more likely that the expert is uninformed about the state when the expert does not disclose information. Therefore, DM's expected state is higher and $a_{\phi}^{*}$ increases. The increase in $a_{\phi}^{*}$ and the decrease in $k^{*}$ imply that the expert's incentive to acquire information decreases. Therefore, $p^{*}$ decreases.

\section{Optimal Expert}

In this section, we consider the situation where DM faces a pool of experts, each with different cost of withholding information. We ask the following question: which expert should DM choose to acquire and report information, in order to maximize DM's expected utility? As shown in the previous section, an expert with a greater cost of withholding information has a lower likelihood of successfully obtaining information and withholds less acquired information. Therefore, it seems ambiguous whether DM can receive more information from an expert with a greater cost of withholding information and receive a higher expected utility.

To tackle this problem, we write down DM's expected utility when the equilibrium is $\left\{a_{\phi}^{*}, p^{*}, k^{*}\right\}$. There are several cases to consider. With probability $p^{*} k^{*}$, the expert obtains information successfully and the observed state is lower than $k^{*}$. The expert withholds the information and DM takes decision $a_{\phi}^{*}$. In this case, the state follows a uniform distribution on $\left[0, k^{*}\right]$ and DM's expected utility is $E_{\theta}\left[-\left(a_{\phi}^{*}-\right.\right.$ $\left.\theta)^{2} \mid \theta \leq k^{*}\right]$. With probability $p^{*}\left(1-k^{*}\right)$, the expert obtains information successfully and the observed state is higher than $k^{*}$. The expert discloses the observed state to DM and DM takes a decision equal to the state. In this case, DM's expected utility is zero. With probability $1-p^{*}$, the expert does not obtain information about the state and DM takes decision $a_{\phi}^{*}$. In this case, the state follows a uniform distribution on $[0,1]$ and DM's expected utility is $E_{\theta}\left[-\left(a_{\phi}^{*}-\theta\right)^{2}\right]$.

From an ex ante point of view, the expected utility of DM when the expert has withholding cost $x$ is

$$
\begin{aligned}
E U_{\mathrm{DM}}(x) \equiv & p^{*} k^{*} E_{\theta}\left[-\left(a_{\phi}^{*}-\theta\right)^{2} \mid \theta \leq k^{*}\right] \\
& +\left(1-p^{*}\right) E_{\theta}\left[-\left(a_{\phi}^{*}-\theta\right)^{2}\right],
\end{aligned}
$$

where the right-hand side can be calculated as

$$
\begin{gathered}
p^{*} k^{*}\left[-\left(a_{\phi}^{*}\right)^{2}+a_{\phi}^{*} k^{*}-\frac{\left(k^{*}\right)^{2}}{3}\right] \\
+\left(1-p^{*}\right)\left[-\left(a_{\phi}^{*}\right)^{2}+a_{\phi}^{*}-\frac{1}{3}\right] .
\end{gathered}
$$

Using numerical simulation, we can get the relationship between DM's ex ante expected utility and the expert's cost of withholding information. This is shown in Figure 3. The figure shows that the ex ante expected utility of DM reaches its highest level when the expert's cost of withholding information is zero.

\section{An Alternative Utility Function of Expert}

In this section, we consider an alternative utility function of the expert. In particular, we assume that when the decision is $a$ and the state is $\theta$, the expert's utility is

$$
u_{E}(a, \theta)=-(a-\theta-b)^{2},
$$

where $b>0$.

When the state is $\theta$, the decision that leads to the highest utility of the expert is $\theta+b$. Therefore, it is no longer true that the expert always prefers a higher decision to a lower one regardless of the state. The expert may prefer a lower decision to a higher one when the state is low. This reflects that 


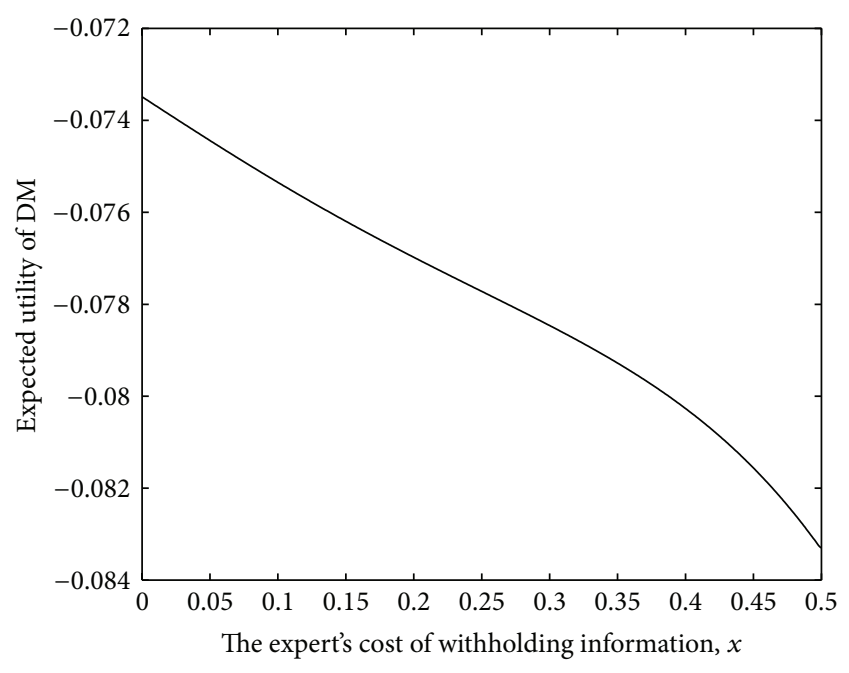

FIGURE 3: How the expected utility of DM changes as $x$ changes.

the expert also has some concerns with the appropriateness of the decision to the underlying state and the conflict of interests between the expert and DM is not as large as in the benchmark case. However, given any state, the decision preferred by the expert is always higher than the one preferred by DM by a constant distance $b$. This reflects that the expert has a bias towards higher decisions by DM.

We characterize a perfect Bayesian Nash equilibrium between DM and the expert in a way similar to what we did before. Denote the decision taken by DM as $a_{\phi}$ when the expert does not disclose information. We consider the expert's optimal strategy in information disclosure when the observed state is $\theta$. If the expert discloses the information, DM will take a decision equal to the disclosed state and the expert's utility is $-b^{2}$. If the expert withholds the information, DM will take decision $a_{\phi}$ and the expert's utility (net of withholding cost $)$ is $-\left(a_{\phi}-\theta-b\right)^{2}-x$. By comparing $-b^{2}$ with $-(a-\theta-b)^{2}-x$, the expert's optimal strategy in information disclosure is to withhold information if and only if the observed state satisfies that

$$
\theta \in\left[l\left(a_{\phi}, b, x\right), h\left(a_{\phi}, b, x\right)\right],
$$

where $l(a, b, x) \equiv(a-b)-\sqrt{b^{2}-x}$ and $h(a, b, x) \equiv(a-$ $b)+\sqrt{b^{2}-x}$. We restrict attention to cases where $b$ is small enough such that $l\left(a_{\phi}, b, x\right) \geq 0$.

Given the expert's optimal strategy in information disclosure, when the effort exerted by the expert in information acquisition $\operatorname{costs} c(p)$, the expert's expected utility is

$$
\begin{aligned}
& 2 p \sqrt{b^{2}-x} E_{\theta}\left[-\left(a_{\phi}-\theta-b\right)^{2}\right.\left.-x \mid l\left(a_{\phi}, b, x\right) \leq \theta \leq h\left(a_{\phi}, b, x\right)\right] \\
&+ p\left(1-2 \sqrt{b^{2}-x}\right)\left(-b^{2}\right) \\
&+(1-p) E_{\theta}\left[-\left(a_{\phi}-\theta-b\right)^{2}\right]-c(p),
\end{aligned}
$$

which can be rewritten as

$$
\begin{aligned}
& 2 p \sqrt{b^{2}-x}\left[-\frac{1}{3\left(b^{2}-x\right)}-x\right] \\
& \quad+p\left(1-2 \sqrt{b^{2}-x}\right)\left(-b^{2}\right) \\
& \quad+(1-p)\left[-\left(a_{\phi}-b\right)^{2}+\left(a_{\phi}-b\right)-\frac{1}{3}\right]-c(p) .
\end{aligned}
$$

The probability $p$ that maximizes the above expected utility satisfies

$$
c^{\prime}(p)=f\left(a_{\phi}, b, x\right),
$$

where

$$
\begin{aligned}
f(a, b, x) \equiv & 2 \sqrt{b^{2}-x}\left[-\frac{1}{3\left(b^{2}-x\right)}-x\right] \\
& +\left(1-2 \sqrt{b^{2}-x}\right)\left(-b^{2}\right) \\
& -\left[-(a-b)^{2}+(a-b)-\frac{1}{3}\right] .
\end{aligned}
$$

For DM, suppose that the expert obtains information with probability $p$, and when successfully obtaining information, the expert withholds information if the observed state $\theta$ satisfies $k_{1} \leq \theta \leq k_{2}$. By Bayes' rule, DM's posterior belief about the state when the expert does not disclose information is

$F(\theta \mid$ No disclosure $)$

$$
=\left\{\begin{array}{cc}
\frac{1-p}{1-p+p\left(k_{2}-k_{1}\right)} \theta, & \text { if } 0 \leq \theta \leq k_{1}, \\
\frac{p\left(k_{2}-k_{1}\right)}{1-p+p\left(k_{2}-k_{1}\right)} \frac{\theta-k_{1}}{k_{2}-k_{1}} & \\
+\frac{1-p}{1-p+p\left(k_{2}-k_{1}\right)} \theta, & \text { if } k_{1}<\theta \leq k_{2}, \\
\frac{p\left(k_{2}-k_{1}\right)}{1-p+p\left(k_{2}-k_{1}\right)} & \\
+\frac{1-p}{1-p+p\left(k_{2}-k_{1}\right)} \theta, & \text { if } k_{2}<\theta \leq 1 .
\end{array}\right.
$$

Under this belief, the optimal decision of DM when the expert does not disclose information is

$$
a_{\phi}=E_{\theta}[\theta \mid \text { No disclosure }]=g\left(p, k_{1}, k_{2}\right),
$$

where

$$
\begin{aligned}
g\left(p, k_{1}, k_{2}\right) \equiv & \frac{p\left(k_{2}-k_{1}\right)}{1-p+p\left(k_{2}-k_{1}\right)} \frac{k_{1}+k_{2}}{2} \\
& +\frac{1-p}{1-p+p\left(k_{2}-k_{1}\right)} \frac{1}{2} .
\end{aligned}
$$

A perfect Bayesian Nash equilibrium is a tuple $\left\{a_{\phi}^{*}, k_{1}^{*}, k_{2}^{*}, p^{*}\right\}$ such that $a_{\phi}^{*}=g\left(p^{*}, k_{1}^{*}, k_{2}^{*}\right), c^{\prime}\left(p^{*}\right)=$ $f\left(a_{\phi}^{*}, b, x\right), k_{1}^{*}=l\left(a_{\phi}^{*}, b, x\right)$, and $k_{2}^{*}=h\left(a_{\phi}^{*}, b, x\right)$. 


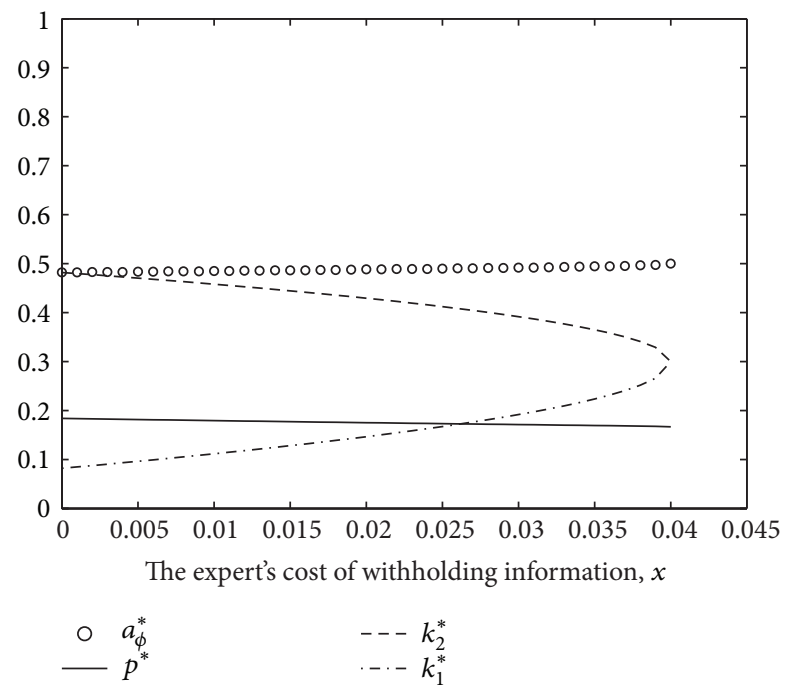

Figure 4: How equilibrium variables change as $x$ changes $(b=0.2)$.

Figure 4 shows the relationship between the cost of withholding information, $x$, and equilibrium variables $a_{\phi}^{*}, p^{*}$, $k_{1}^{*}$, and $k_{2}^{*}$ when $b=0.2$. From the figure, we can see that, in equilibrium, the expert's effort in acquiring information decreases as $x$ increases. This result indicates a negative effect of the cost of withholding information on the expert's incentive to acquire information. However, the negative effect is much smaller than in the benchmark case. This can be seen from Figure 4 that the expert's effort in acquiring information approaches a positive value as $x$ approaches $b^{2}$ from below, even though the expert discloses all information $\left(k_{2}^{*}\right.$ and $k_{1}^{*}$ approach each other). This can also be seen from the fact that $f\left(a_{\phi}, b, x\right)>0$ when $x=b^{2}$.

As the next step, we consider a situation where DM faces a pool of experts with different costs of withholding information. We only consider the case where the cost of withholding information is small; in particular, $x \leq b^{2}$. This is because when $x>b^{2}$, the expert will disclose all information and the cost of withholding information does not affect the expert's strategy in information acquisition. From an ex ante point of view, DM's expected utility by choosing an expert with cost $x$ of withholding information is

$$
\begin{aligned}
& p^{*}\left(k_{2}^{*}-k_{1}^{*}\right) E_{\theta}\left[-\left(a_{\phi}^{*}-\theta\right)^{2} \mid k_{1}^{*} \leq \theta \leq k_{2}^{*}\right] \\
& +\left(1-p^{*}\right) E_{\theta}\left[-\left(a_{\phi}^{*}-\theta\right)^{2}\right],
\end{aligned}
$$

which could be calculated as

$$
\begin{aligned}
& -p^{*}\left(a_{\phi}^{*}\right)^{2}\left(k_{2}^{*}-k_{1}^{*}\right)+p^{*} a_{\phi}^{*}\left[\left(k_{2}^{*}\right)^{2}-\left(k_{1}^{*}\right)^{2}\right] \\
& -\frac{1}{3 p^{*}\left[\left(k_{2}^{*}\right)^{3}-\left(k_{1}^{*}\right)^{3}\right]}+\left(1-p^{*}\right)\left[-\left(a_{\phi}^{*}\right)^{2}+a_{\phi}^{*}-\frac{1}{3}\right] .
\end{aligned}
$$

For a fixed bias $b$ of the expert, we use numerical simulation to identify the relationship between DM's ex

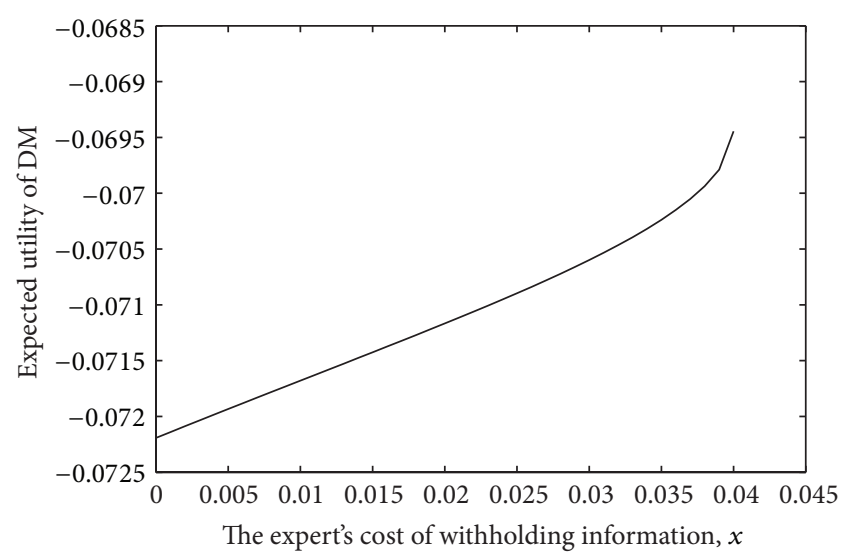

FIGURE 5: How the expected utility of DM changes as $x$ changes $(b=$ $0.2)$.

ante expected utility and the expert's cost of withholding information. Figure 5 shows this relationship when $b=0.2$ and $x \leq b^{2}$. It can be seen from the figure that DM's expected utility reaches its maximum level when the expert's withholding cost is at its largest value.

\section{Conclusion}

This paper studies a game between an expert and a decision maker. The expert first exerts costly effort to acquire decisionrelevant information. Then the expert decides whether or not to disclose the acquired information to the decision maker. Finally, the decision maker takes a decision that affects the utilities of both the expert and the decision maker. The expert and the decision maker have different preferences about the decision. In addition, withholding information is costly to the expert. We find that an expert with a greater withholding cost exerts less effort to acquire information but discloses a greater proportion of acquired information to DM. When the expert prefers DM's decision to be as high as possible, DM's expected utility decreases when the expert's withholding cost increases. When the decision preferred by the expert is higher than that preferred by DM by a constant distance, DM's expected utility increases when the expert's withholding cost increases.

Future work involves studying similar games with other utility functions of the expert. Another direction for future studies is to analyze a model where the expert can misreport the observed state but will incur a cost when doing so. In such a model, we can investigate the relationship between the expert's cost of misreporting information and the expert's incentive to acquire information. This model is more complicated than the one we examine in this paper and is left for future research.

\section{Conflict of Interests}

The author declares that there is no conflict of interests regarding the publication of this paper. 


\section{Acknowledgment}

This research is supported by the Fundamental Research Funds for the Central Universities (no. JBK140107).

\section{References}

[1] S. J. Grossman, "The informational role of warranties and private disclosure about product quality," Journal of Law and Economics, vol. 24, no. 3, pp. 461-483, 1981.

[2] S. J. Grossman and O. Hart, "Disclosure laws and takeover bids," The Journal of Finance, vol. 35, no. 2, pp. 323-334, 1980.

[3] P. Milgrom, "Good news and bad news: representation theorems and applications," Bell Journal of Economics, vol. 12, no. 2, pp. 380-391, 1981.

[4] P. Milgrom and J. Roberts, "Relying on the information of interested parties," The RAND Journal of Economics, vol. 17, no. 1, pp. 18-32, 1986.

[5] H. S. Shin, "News management and the value of firms," The RAND Journal of Economics, vol. 25, no. 1, pp. 58-71, 1994.

[6] H. S. Shin, "Adversarial and inquisitorial procedures in arbitration," RAND Journal of Economics, vol. 29, no. 2, pp. 378-405, 1998.

[7] Y.-K. Che and N. Kartik, "Opinions as incentives," Journal of Political Economy, vol. 117, no. 5, pp. 815-860, 2009.

[8] F. Xu and W. Suen, "Investment in concealable information," working paper, University of Hong Kong, 2012. 


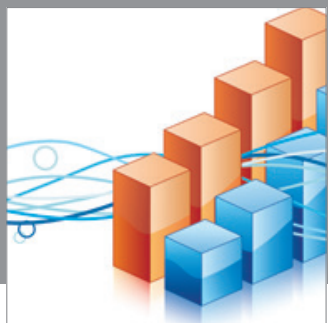

Advances in

Operations Research

mansans

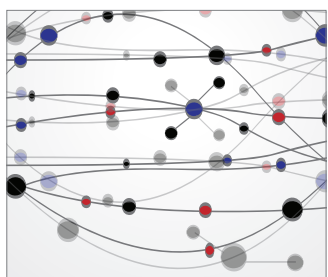

The Scientific World Journal
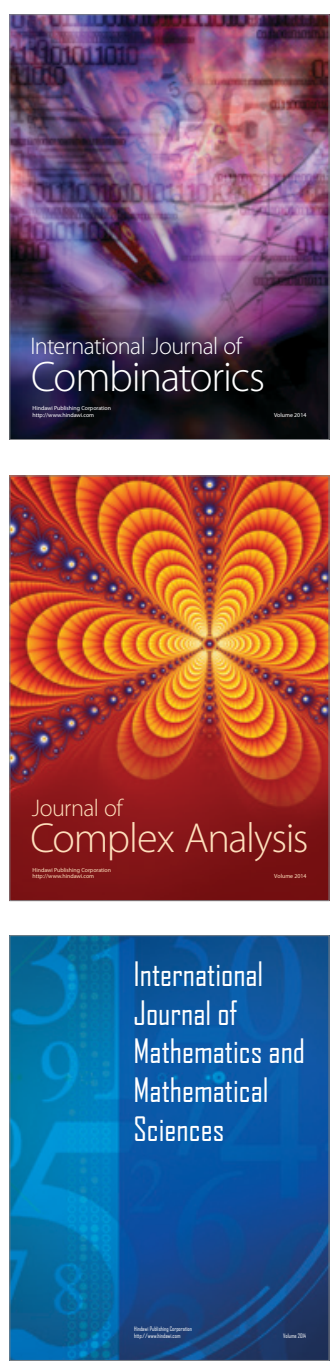
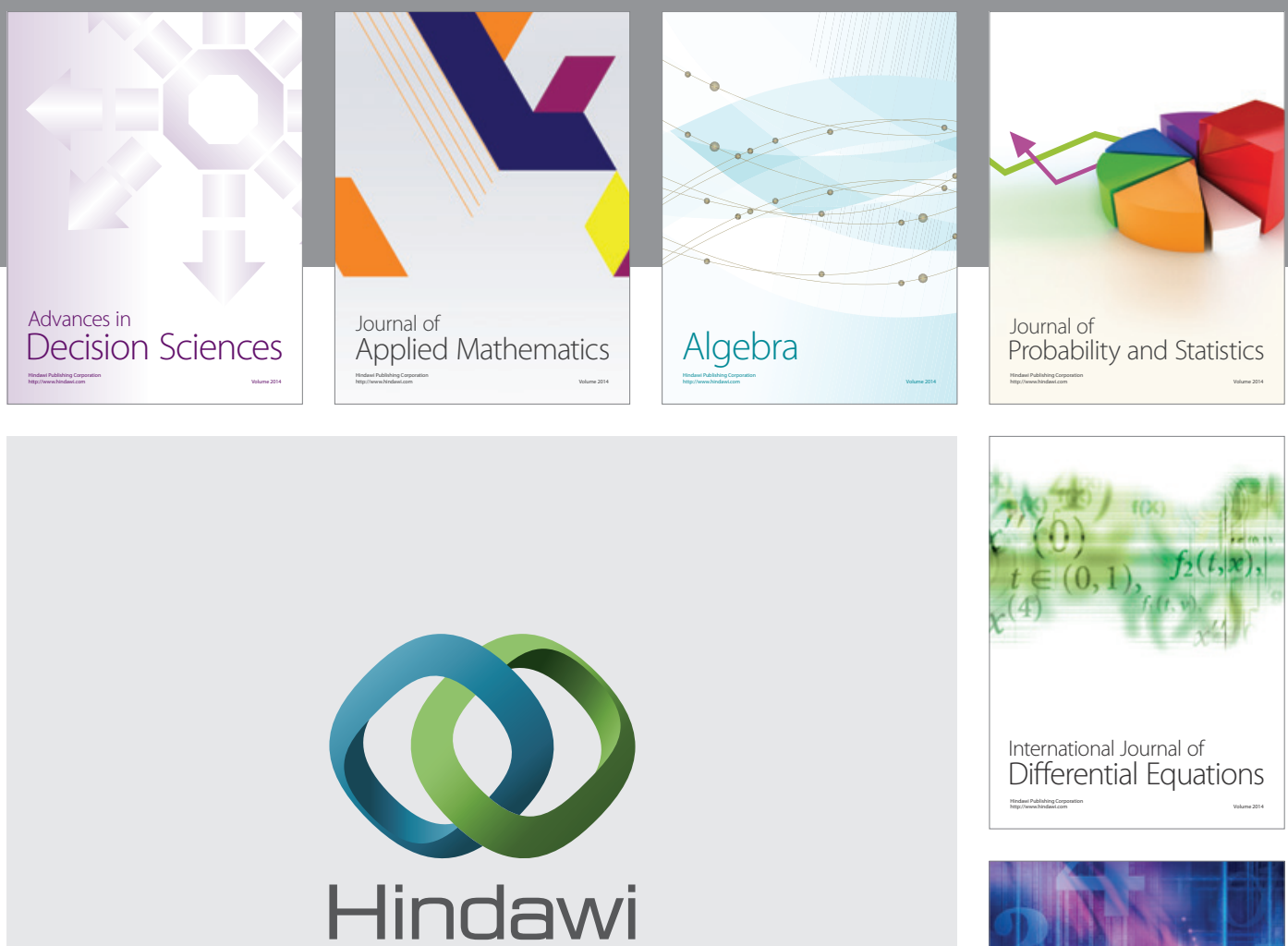

Submit your manuscripts at http://www.hindawi.com
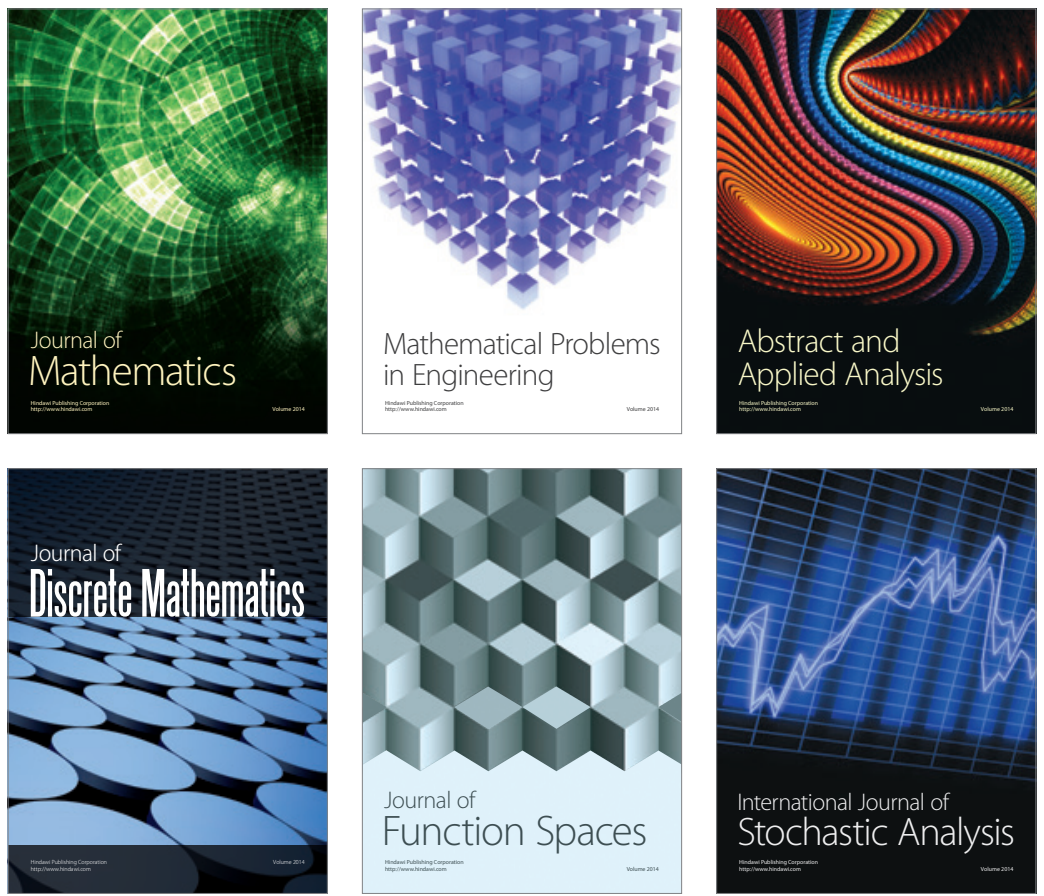

Journal of

Function Spaces

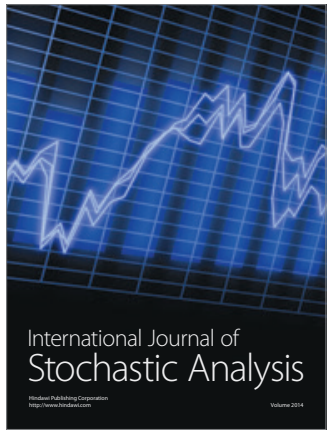

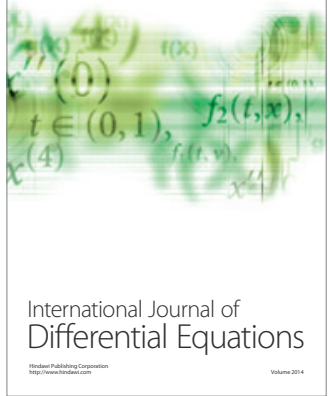
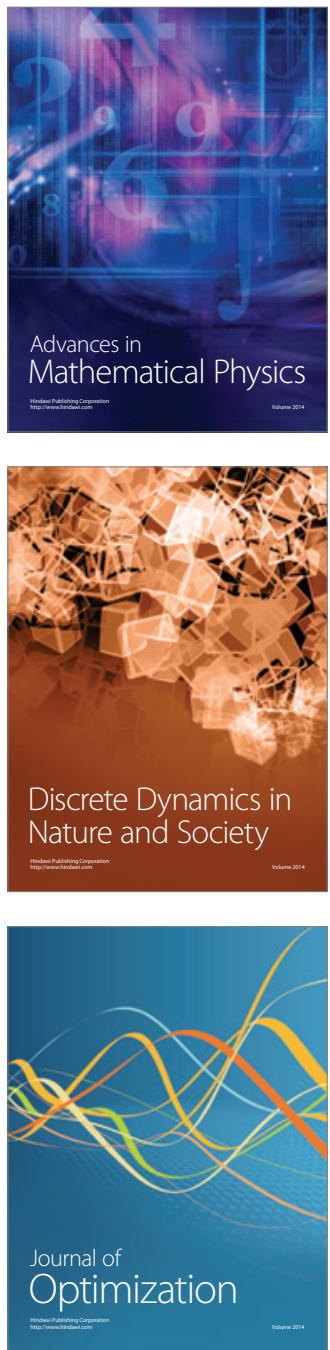